\title{
On the Uniqueness of the Equilibrium State for Plane Rotators
}

\author{
J. Bricmont and J. R. Fontaine \\ Institut de Physique Théorique, U.C.L., Université de Louvain, B-1348 Louvain-la-Neuve, Belgium \\ L. J. Landau \\ Department of Mathematics, Bedford College, London NW1 4NS, England
}

\begin{abstract}
We study the classical statistical mechanics of the plane rotator, and show that there is a unique translation invariant equilibrium state in zero external field, if there is no spontaneous magnetization. Moreover, this state is then extremal in the equilibrium states. In particular there is a unique phase for the two dimensional rotator, and a unique phase for the three dimensional rotator above the critical temperature. It is also shown that in a sufficiently large external field the Lee-Yang theorem implies uniqueness of the equilibrium state.
\end{abstract}

\section{Introduction}

Some new results have been obtained recently concerning the classical statistical mechanics of the plane rotator model, defined by the Hamiltonian

$$
H=-\beta \sum J_{i j} \sigma_{i} \cdot \sigma_{j} \quad J_{i j} \geqq 0
$$

where $\sigma_{i}$ is a two-dimensional vector of unit length. For $d \geqq 3$, it has been proven [6] that spontaneous magnetization occurs for large $\beta$. For $d=2$, it is well-known that there is no spontaneous magnetization for sufficiently short range interactions [17]; moreover Dobrushin and Shlosman have proven that for finite range interactions all the equilibrium states are invariant under the action of $S O(2)$ on the configuration space [2]. One may ask: does the absence of spontaneous magnetization imply uniqueness of the equilibrium state, as for example in the Ising model $[16,19]$ ? We show in the present paper that it implies at least uniqueness of the translation invariant equilibrium state and that the latter cannot be decomposed into non-invariant equilibrium states. Compared with the similar problem in the Ising model $[16,19]$, our method looks more complicated; the reason is that for this model, we don't have correlation inequalities comparable to the F.K.G. inequalities [5] which are valid for all the different boundary conditions. Instead of considering boundary conditions, we characterize invariant equilibrium states as tangents to the pressure [9]. We introduce various perturbations to the pressure and control the derivatives of these perturbed pressures with correlation inequalities. 
In a sufficiently large external field, the correlation inequalities are sufficient to control all boundary conditions, and together with the Lee-Yang theorem gives uniqueness of the equilibrium state.

The extension to models where $\sigma_{i}$ has more than two components seems difficult because of the lack of the necessary correlation inequalities.

Acknowledgements. It is a pleasure to thank F. Dunlop and J. Slawny for helpful discussions, and A. Messager, S. Miracle, and Ch. E. Pfister for providing us with Lemma 3.2 [24] which leads to a simplification of our original proof of the extremality of the unique phase.

\section{The Model}

At each point $i$ of the lattice $\mathbb{Z}^{d}$ is associated a spin variable $\sigma_{i} \in \mathbb{R}^{2}$. We use two parametrizations, given by:

$$
\sigma_{i}=\left(s_{i}, t_{i}\right)=\left(r_{i} \cos \phi_{i}, r_{i} \sin \phi_{i}\right)
$$

where

$$
s_{i}, t_{i} \in \mathbb{R}, \quad r_{i} \in \mathbb{R}_{+}, \quad \phi_{i} \in[0,2 \pi[.
$$

The a priori probability distribution for $\sigma_{i}$ is assumed rotation invariant:

$$
d v_{0}\left(\boldsymbol{\sigma}_{i}\right)=d \lambda\left(r_{i}\right) d \phi_{i}
$$

and the spin is bounded: $\left|\boldsymbol{\sigma}_{i}\right| \leqq b$ with probability 1 . In addition the measure $v_{0}$ satisfies Condition A, which is important for the development of correlation inequalities:

\section{Condition A.}

$$
\begin{aligned}
& \forall \alpha, \beta, \gamma, \delta \in \mathbb{N} \\
& \int\left(s+s^{\prime}\right)^{\alpha}\left(s-s^{\prime}\right)^{\beta}\left(t+t^{\prime}\right)^{\gamma}\left(t^{\prime}-t\right)^{\delta} d v_{0}(s, t) d v_{0}\left(s^{\prime}, t^{\prime}\right) \geqq 0 .
\end{aligned}
$$

Remark. 1) The following measures (suitably normalized) are known to satisfy Condition A $[1,3,12,18,22]$ :
i) $\delta(r-b) d r d \phi \quad b>0 \quad$ (fixed length)
ii) $\chi(r \in[0, b]) r d r d \phi \quad b>0 \quad$ (uniform distribution)
iii) $\exp \left(-a r^{4}+c r^{2}\right) \chi(r \in[0, b]) r d r d \phi \quad a, b>0$.

The interaction between spins is given by a translation invariant, ferromagnetic pair interaction

where

$$
H=-\sum J_{i j} \sigma_{i} \cdot \sigma_{j}
$$

$$
J_{i j}=J(i-j) \geqq 0 .
$$

We consider the case of finite range interactions:

$$
J_{i j}=0 \text { if }|i-j|>L .
$$

However our results are not sensitive to this restriction and carry over to long range interactions with sufficiently rapid fall-off. 
If $\Lambda$ is a finite region, an exterior configuration $\omega$ is a specification of the spins in $\Lambda_{c}$, the complement of $\Lambda$, and a boundary condition is a probability measure $\mu_{b c}(\omega)$ on the exterior configurations. A particularly useful boundary condition is denoted $s-$ b.c. for which all spins in $\Lambda_{c}$ are in the $s$-direction with maximum value:

$$
\text { for all } i \in \Lambda_{c} \quad \sigma_{i}=\left(s_{i}=\max _{\operatorname{supp} \lambda} r, t_{i}=0\right) \text {. }
$$

The boundary condition $t$-b.c. is obtained by the interchange of $s$ and $t$ variables.

An exterior configuration $\omega$ induces an effective Hamiltonian for the region $\Lambda$ given by

$$
H_{\Lambda, \omega}=-\sum_{i, j \in \Lambda} J_{i j} \sigma_{i} \cdot \sigma_{j}-\sum_{\substack{i \in \Lambda \\ j \in A_{c}}} J_{i j} \sigma_{i} \cdot \sigma_{j}(\omega)
$$

where $\sigma_{j}(\omega)$ takes the value specified by $\omega$.

The joint probability distribution of the spins in the region $\Lambda$ is given by the Boltzmann factor

$$
d \mu_{\Lambda, \omega}=Z_{\Lambda, \omega}^{-1} \exp \left(-H_{\Lambda, \omega}\right) \prod_{i \in \Lambda} d v_{0}\left(\boldsymbol{\sigma}_{i}\right)
$$

where

$$
Z_{\Lambda, \omega}=\int \exp \left(-H_{\Lambda, \omega}\right) \prod_{i \in \Lambda} d v_{0}\left(\sigma_{i}\right) .
$$

Expectations with respect to $\mu_{\Lambda, \omega}$ will be denoted \langle\rangle$_{\Lambda, \omega}$. For a general boundary condition

$$
\langle\rangle_{A, b c}=\int\langle\rangle_{A, \omega} \mathrm{d} \mu_{b c}(\omega) .
$$

The pressure

$$
p_{\Lambda, \omega}=|\Lambda|^{-1} \ln Z_{\Lambda, \omega}
$$

and for a general boundary condition

$$
p_{\Lambda, b c}=\int p_{\Lambda, \omega} d \mu_{b c}(\omega) .
$$

The pressure

$$
p=\lim _{\Lambda \rtimes \mathbb{Z}^{d}} p_{\Lambda, b c}
$$

and is independent of the boundary condition [20].

An equilibrium state is an infinite volume limit of finite region states \langle\rangle$_{A, b c}$ with some boundary condition, or equivalently a probability measure on the infinite volume configuration space satisfying the DLR equations $[9,13]$. We will generally use the symbol $<>$ for a finite region state and the symbol $\varrho$ for an infinite volume equilibrium state. The set of equilibrium states for the Hamiltonian $H$ is denoted $\Delta(H)$. A phase is an equilibrium state invariant under the lattice translations, and the set of phases is denoted $\Delta_{I}(H)$.

In an external field $h \boldsymbol{n}$ the Hamiltonian (2.1) is modified to

$$
\begin{aligned}
H_{h} & =-\sum J_{i j} \boldsymbol{\sigma}_{i} \cdot \boldsymbol{\sigma}_{j}-\sum h \boldsymbol{n} \cdot \boldsymbol{\sigma}_{i} \\
& =-\sum J_{i j}\left(s_{i} s_{j}+t_{i} t_{j}\right)-\sum\left(h_{s} s_{i}+h_{t} t_{i}\right) .
\end{aligned}
$$


Definition. The spontaneous magnetization $M$ is defined by

$$
M=\lim _{h \downarrow 0} \varrho_{h}\left(\boldsymbol{n} \cdot \boldsymbol{\sigma}_{i}\right)
$$

where

$$
\varrho_{h} \in \Delta_{I}\left(H_{h}\right) .
$$

Remark. By translation invariance of the phase $\varrho_{h}, M$ is independent of $i$; by rotation covariance, $M$ is independent of the direction $\boldsymbol{n}$. Using the fact that the pressure is a convex function of $h$ and the relation between phases and tangents to the graph of the pressure [9] it is seen that $M$ is the right-derivative of the pressure with respect to $h$ at $h=0$ and is independent of the choice of phase $\varrho_{h} \in \Delta_{I}\left(H_{h}\right)$.

Our basic results can now be formulated:

2.1. Theorem. If the spontaneous magnetization $M=0$ then there is a unique phase in zero external field. Moreover, this phase is then extremal in the equilibrium states. This phase is also the unique quasi-periodic state.

2.2. Theorem. Let $d \lambda(r)=\delta(r-b) d r$. Then for a large enough external field the equilibrium state is unique.

2.3. Corollary. If the lattice dimension $d=2$, there is a unique phase in zero external field. If $d=3$, there is a unique phase for $T>T_{c}$ where $T_{c}$ is the critical temperature for spontaneous magnetization.

We point out that for simplicity of presentation we have not given each theorem or lemma in its most general form. We discuss extensions in Section 6.

\section{Inequalities}

Let $A$ be a finite subset of $\mathbb{Z}^{d}$ with multiplicities, and define

$$
s_{A}=\prod_{i \in A} s_{i} .
$$

For example $s_{i}^{2} s_{j}=s_{A}$ where $A=\{i, i, j\}$.

$|A|$ denotes the (finite) cardinality of $A$. Similarly we define $t_{A}$ and $r_{A}$. If $N$ is a function from $\mathbb{Z}^{d}$ to $\mathbb{Z}$ which is equal to zero except at a finite number of points, we define

$$
N \phi=\sum n_{i} \phi_{i}
$$

Note that if $|A|$ is even, $s_{A} \pm t_{A}$ may be expressed in terms of cosines.

$$
s_{A}+t_{A}=2^{(1-|A|)} r_{A} \sum_{M} \cos M \phi
$$

and similarly for $s_{A}-t_{A}$ : We decompose $s_{A} \pm t_{A}$ into a sum of products of $r_{i} r_{j}$ $\left(\cos \phi_{i} \cos \phi_{j} \pm \sin \phi_{i} \sin \phi_{j}\right)=r_{i} r_{j} \cos \left(\phi_{i} \mp \phi_{j}\right)$ and then use the identity $\cos N \phi \cos M \phi=\frac{1}{2}[\cos (N+M) \phi+\cos (N-M) \phi]$. 
We denote by $\operatorname{supp} A$ the set $A$ without multiplicities:

$$
\operatorname{supp} A=\left\{i \in \mathbb{Z}^{d}: i \in A\right\} \text {. }
$$

Also

$$
\operatorname{supp} N=\left\{i \in \mathbb{Z}^{d}: n_{i} \neq 0\right\} .
$$

We define the algebra $\mathfrak{A}$ as the set of finite linear combinations of the functions $s_{A} t_{B}$, and the even and odd subspaces $\mathfrak{A}_{e}, \mathfrak{H}_{0}$ as linear combinations of $\left\{r_{A} \cos N \phi\right\}$, respectively $\left\{r_{A} \sin N \phi\right\}$. Equivalently these subspaces are defined as linear combinations of $\left\{s_{A} t_{B}\right\}$ with $|B|$ even, respectively odd. The subspaces may equally well be defined according to transformation properties under the transformation $t_{i} \rightarrow-t_{i} \forall i\left(\phi_{i} \rightarrow-\phi_{i} \forall i\right)$. Consider the interaction (2.2) with the external field in the positive $s$-direction. The Hamiltonian has the form

$$
H=-\sum J_{i j} r_{i} r_{j} \cos \left(\phi_{i}-\phi_{j}\right)-h \sum r_{i} \cos \phi_{i} .
$$

With s-b.c. the effective interaction in the region $\Lambda$ has the form

$$
H_{A, s}=-\sum_{i, j \in A} J_{i j} r_{i} r_{j} \cos \left(\phi_{i}-\phi_{j}\right)-\sum_{i \in A} \alpha_{i} r_{i} \cos \phi_{i}
$$

where $\alpha_{i} \geqq 0$. Then Ginibre's inequalities hold [Appendix, Theorems A1, A4]

$$
\left\langle r_{A} \cos N \phi r_{B} \cos M \phi\right\rangle_{s, \Lambda} \geqq\left\langle r_{A} \cos N \phi\right\rangle_{s, A}\left\langle r_{B} \cos M \phi\right\rangle_{s, \Lambda} .
$$

Now consider the interaction (2.2) with the external field such that $h_{s}, h_{t} \geqq 0$. Then the generalized Griffiths' inequalities hold [Theorems A2, A3]

$$
\begin{aligned}
&\left\langle s_{A} s_{B}\right\rangle_{s, A} \geqq\left\langle s_{A}\right\rangle_{s, A}\left\langle s_{B}\right\rangle_{s, A} \\
&\left\langle t_{A} t_{B}\right\rangle_{s, A} \geqq\left\langle t_{A}\right\rangle_{s, A}\left\langle t_{B}\right\rangle_{s, A} \\
& 0 \leqq\left\langle s_{A} t_{B}\right\rangle_{s, A} \leqq\left\langle s_{A}\right\rangle_{s, A}\left\langle t_{B}\right\rangle_{s, A} .
\end{aligned}
$$

In addition there are comparison inequalities relating different states [Theorems A3, A4].

We derive here some basic results which will be useful in the proofs of Theorems 2.1 and 2.2. The discussion will be given for the interaction (2.1).

3.1. Theorem. $\varrho_{s} \equiv \lim _{\Lambda>\mathbb{Z}^{d}}\langle\rangle_{s, \Lambda}$ exists by monotonicity and is an extremal equilibrium state.

Remark. We note a useful property of the state $\varrho_{s}$. By monotonicity in both $h$ and $\Lambda$ it follows that $M=\varrho_{s}\left(s_{i}\right)$.

The proof of Theorem 3.1 is based on ${ }^{1}$.

3.2. Lemma. Let $\omega$ be any exterior configuration for the region $\Lambda$. Then

$$
\left\langle r_{A} \cos M \phi\right\rangle_{s, \Lambda} \geqq\left\langle r_{A} \cos M \phi\right\rangle_{\omega, A} .
$$

Proof. We must show

$$
\int\left(r_{A} \cos N \phi-r_{A}^{\prime} \cos N \phi^{\prime}\right) d \mu_{s}\left(\left\{r_{i}, \phi_{i}\right\}\right) d \mu_{\omega}\left(\left\{r_{i}^{\prime}, \phi_{i}^{\prime}\right\}\right) \geqq 0 .
$$

1 Lemma 3.2 is due to A. Messager, S. Miracle, and Ch. E.Pfister. It simplifies our original proof of Theorem 3.1 
This is proved in the same way as Ginibre's inequalities [7] once the effective Hamiltonians are combined as

$$
\begin{aligned}
-H_{\Lambda, s}-H_{\Lambda, \omega}= & \sum J_{i j}\left[r_{i} r_{j} \cos \left(\phi_{i}-\phi_{j}\right)+r_{i}^{\prime} r_{j}^{\prime} \cos \left(\phi_{i}^{\prime}-\phi_{j}^{\prime}\right)\right] \\
& +\sum \alpha_{i} r_{i} \cos \phi_{i}+\alpha_{i}^{\prime} r_{i}^{\prime} \cos \left(\phi_{i}^{\prime}+\psi_{i}\right)
\end{aligned}
$$

where

$$
\left|\alpha_{i}^{\prime}\right| \leqq \alpha_{i}
$$

This can be written as a sum with positive coefficients of products of $\left(r_{i} \pm r_{i}^{\prime}\right)$, $\cos \left(\frac{\phi_{i}^{\prime}+\phi_{i}+\psi}{2}\right) \cos \left(\frac{\phi_{i}^{\prime}-\phi_{i}+\psi}{2}\right)$ and similar terms with sine instead of cosine. $\left(r_{A} \cos M \phi-r_{A}^{\prime} \cos M \phi^{\prime}\right)$ can be written similarly.

Then one expands the exponential in series and, due to the above decompositions, each term is a product of integrals over $r_{i}, r_{i}^{\prime}$ and over $\phi_{i}, \phi_{i}^{\prime}$. The integral over $\phi_{i}, \phi_{i}^{\prime}$ factorizes into a product of two integrals of two identical functions, one in the variables $\left(\phi_{i}^{\prime}+\phi_{i}\right)$ and the other in $\left(\phi_{i}^{\prime}-\phi_{i}\right)$. Being a square, this expression is positive. The integral over the variables $r_{i}, r_{i}^{\prime}$ reduces to a product of integrals of the type:

$$
\int\left(r_{i}+r_{i}^{\prime}\right)^{a_{i}}\left(r_{i}-r_{i}^{\prime}\right)^{b_{i}} d \lambda_{i}\left(r_{i}\right) d \lambda_{i}\left(r_{i}^{\prime}\right)
$$

This integral obviously vanishes if $b_{i}$ is odd and is positive if $b_{i}$ is even.

Definition. We say the state $\varrho$ is clustering if for all $f, g \in \mathfrak{A}$

$$
\varrho\left(f \tau_{a} g\right)-\varrho(f) \varrho\left(\tau_{a} g\right) \rightarrow 0 \quad \text { as } \quad|a| \rightarrow \infty
$$

where $\tau_{a}$ denotes a translation by the amount $a$.

Proof of Theorem 3.1. From Lemma 3.2 it follows that ${ }^{2}\left\langle r_{A} \cos M \phi\right\rangle_{s, \Lambda}(\geqq 0)$ decreases as $\Lambda \nearrow \mathbb{Z}^{d}$. Thus the limit exists. Since $\left\langle r_{A} \sin M \phi\right\rangle_{s, \Lambda}=0$ the state $\varrho_{s}$ is welldefined. Again from Lemma 3.2 it follows that

$$
\varrho\left(r_{A} \cos N \phi\right) \leqq \varrho_{s}\left(r_{A} \cos N \phi\right) \text { for all } \varrho \in \Delta(H),
$$

which shows that $\varrho_{s}$ is extremal as a state on the even subspace $\mathfrak{U}_{e}$.

The extremality of $\varrho_{s}$ on the even subspace $\mathfrak{A}_{e}$ implies that $\varrho_{s}$ is clustering on $\mathfrak{A}_{e}$. Indeed, since $\Delta(H)$ is a metrizable Choquet simplex [9], there is a unique probability measure $\omega_{s}$ carried by the extremal elements of $\Delta(H)$ such that

$$
\varrho_{s}(f)=\int \sigma(f) d \omega_{s}(\sigma), \quad \forall f \in \mathfrak{U} .
$$

By Lemma 3.2 it follows that $\varrho_{s} \uparrow \mathfrak{U}_{e}=\sigma \uparrow \mathfrak{U}_{e} \omega_{s}$-a.e. Since $\sigma$ is clustering, $\varrho_{s}$ is clustering on $\mathfrak{A}_{e}$. By correlation inequalities it follows that $\varrho_{s}$ is clustering on the full algebra $\mathfrak{A}$ : (Theorem A5 or [3])

$$
\begin{aligned}
\mid \varrho_{s}\left(s_{A} t_{B} s_{C} t_{D}\right) & -\varrho_{s}\left(s_{A} t_{B}\right) \varrho_{s}\left(s_{C} t_{D}\right) \mid \\
& \leqq\left(\varrho_{s}\left(s_{A} s_{B} s_{C} s_{D}\right)-\varrho_{s}\left(s_{A} s_{B}\right) \varrho_{s}\left(s_{C} s_{D}\right)\right) \text { if }|B| \text { is even }
\end{aligned}
$$

and

$$
\varrho_{s}\left(s_{A} t_{B} S_{C} t_{D}\right)^{2} \leqq\left(\varrho_{s}\left(s_{A} S_{B} S_{C} S_{D}\right)^{2}-\varrho_{s}\left(s_{A} S_{B}\right)^{2} \varrho_{s}\left(s_{C} S_{D}\right)^{2}\right) \text { if }|B| \text { is odd }
$$

2 Lemma 3.2 holds for all exterior configurations and hence for any boundary condition $b$. Take $b$ as the boundary condition induced by $s$-b.c. in $\Lambda_{c}^{\prime}$ where $\Lambda^{\prime} \supset \Lambda$ 
By construction, the restriction of $\varrho_{s}$ to the odd subspace $\mathfrak{A}_{0}$ is identically zero. Thus the extremality of $\varrho_{s}$ follows from the following lemma.

3.3. Lemma. (a) Let $\varrho, \sigma$ be two states on $\mathfrak{A}$ satisfying

(i) $\varrho, \sigma$ are clustering;

(ii) $\varrho / \mathfrak{A}_{e}=\sigma / \mathfrak{A}_{e}$;

(iii) $\varrho / \mathfrak{U}_{0}=0$,

then $\lim _{i \rightarrow \infty} \sigma\left(\tau_{i}(f)\right)=0, \forall f \in \mathfrak{U}_{0}$.

(b) Let $\omega$ be a translation invariant probability measure on a set $E$ of states $\sigma$, satisfying $\lim _{i \rightarrow \infty} \sigma\left(\tau_{i}(f)\right)=0$ w-a.e. for some $f \in \mathfrak{A}$.

Then $\sigma(f)=0$ w-a.e.

(c) Let $\varrho=\int_{E} \sigma d \omega(\sigma)$ be a decomposition of the invariant state $\varrho$ into a set of states $E$, with the measure $\omega$ translation invariant. Suppose $\varrho$ and $\omega$-a.e. $\sigma$ satisfy the hypothesis in (a). Then $\varrho=\sigma \omega$-a.e.

Proof. (a) By (i) and (iii)

$$
\lim _{i \rightarrow \infty} \varrho\left(f \tau_{i}(g)\right)=0 \quad \forall f, g \in \mathfrak{U}_{0}
$$

and by (ii)

$$
\lim _{i \rightarrow \infty} \sigma\left(f \tau_{i}(g)\right)=0 .
$$

Therefore by (i) $\sigma(f) \lim _{i \rightarrow \infty} \sigma\left(\tau_{i}(g)\right)=0 \forall f, g \in \mathfrak{A}_{0}$ and the conclusion follows.

(b) Since $\omega$ is translation invariant, $\int_{E}|\sigma(f)| d \omega(\sigma)=\int_{E}\left|\sigma\left(\tau_{i}(f)\right)\right| d \omega(\sigma), \forall i \in \mathbb{Z}^{d}$ and by dominated convergence, $\left(\left|\sigma\left(\tau_{i}(f)\right)\right| \leqq\|f\|\right)$

$$
\lim _{i \rightarrow \infty} \int_{E} \mid \sigma\left(\tau_{i}(f) \mid d \omega(\sigma)=0 .\right.
$$

So $\sigma(f)=0 \omega$-a.e.

(c) By points (a) and (b) $\sigma(f)=0 \omega$-a.e. for each $f \in \mathfrak{A}_{0}$. Since $\mathfrak{A}_{0}$ is separable, we conclude $\sigma / \mathfrak{U}_{0}=0 \omega$-a.e. and $\sigma=\varrho \omega$-a.e.

We now begin the argument leading to the proof of Theorem 2.1.

There is a useful "bootstrap" principle which allows one to conclude that certain higher order correlation functions are zero if certain lower order ones are zero. In particular we shall conclude from $M=0$ and Lemma 3.2 that the equilibrium states have certain symmetry properties.

3.4. Theorem. Let $\varrho$ be any weak* limit point of the finite volume states \langle\rangle$_{\Lambda}$ as $\Lambda \nearrow \mathbb{Z}^{d}$, where the effective Hamiltonian for the region $\Lambda$ has the form

$$
-H_{\Lambda}=\sum_{i, j \in \Lambda} J_{i j}\left(s_{i} s_{j}+t_{i} t_{j}\right)+\sum_{i \in \Lambda} \alpha_{i} s_{i}+\beta_{i} t_{i}
$$

with $\alpha_{i} \geqq\left|\beta_{i}\right|$. 
Then if $\varrho\left(s_{i}-t_{i}\right)=0$ for all $i$, it follows that $\varrho\left(s_{A}-t_{A}\right)=0$ for all $A$.

Proof. Define the random variables $x_{i}=2^{-\frac{1}{2}}\left(s_{i}+t_{i}\right), y_{i}=2^{-\frac{1}{2}}\left(s_{i}-t_{i}\right)$. The effective Hamiltonian in terms of the variables $x, y$ is

$$
\sum J_{i j}\left(x_{i} x_{j}+y_{i} y_{j}\right)+2^{-\frac{1}{2}} \sum\left(\alpha_{i}+\beta_{i}\right) x_{i}+\left(\alpha_{i}-\beta_{i}\right) y_{i} \text {. }
$$

Also the a priori measure for $(x, y)$ is the same as for $(s, t)$ by rotation invariance.

Therefore the generalized Griffiths' inequalities are valid (Theorem A2) and these extend to the limit point $\varrho$ :

$$
0 \leqq \varrho\left(x_{B} y_{C}\right) \leqq \varrho\left(x_{B}\right) \varrho\left(y_{C}\right) .
$$

We first show: Given $n$ (odd), if $\varrho\left(y_{A}\right)=0$ for all $|A|($ odd $)<n$ then $\varrho\left(s_{A}\right)=\varrho\left(t_{A}\right)$ for all $|A|<n$.

Indeed $^{3}$

$$
\varrho\left(s_{A}-t_{A}\right)=2^{1-\frac{1}{2}|A|} \sum_{\substack{B \cup C=A \\|C| \text { odd }}} \varrho\left(x_{B} y_{C}\right)=0 \text { if }|A|<n
$$

by (3.5) and the hypothesis $\varrho\left(y_{C}\right)=0$.

We next show: $\varrho\left(y_{A}\right)=0$ for all $|A|$ odd. Indeed, by hypothesis $\varrho\left(s_{i}\right)=\varrho\left(t_{i}\right)$ for all $i$ and so $\varrho\left(y_{i}\right)=0$ for all $i$. The proof proceeds by induction. Let $n$ (odd) be given and suppose $\varrho\left(y_{A}\right)=0$ for all $|A|$ (odd $)<n$. To show $\varrho\left(y_{A}\right)=0$ if $|A|=n$ we write

$$
\begin{aligned}
\varrho\left(y_{A}\right) & =2^{-\frac{1}{2}|A|} \sum_{B \cup C=A} \varrho\left(s_{B} t_{C}\right)(-1)^{|C|} \\
& =2^{-\frac{1}{2}|A|}\left\{\varrho\left(s_{A}-t_{A}\right)+\sum_{\substack{B \cup C=A \\
|B| \text { odd } \\
|B|<|A|}} \varrho\left(s_{B} t_{C}\right)-\varrho\left(s_{C} t_{B}\right)\right\} .
\end{aligned}
$$

But

$$
\begin{aligned}
\left|\varrho\left(s_{B} t_{C}\right)-\varrho\left(s_{C} t_{B}\right)\right| & =\left|\varrho\left(s_{B} t_{C}\right)-\varrho^{\prime}\left(s_{B} t_{C}\right)\right| \\
& \leqq \varrho\left(s_{B}\right) \varrho^{\prime}\left(t_{C}\right)-\varrho^{\prime}\left(s_{B}\right) \varrho\left(t_{C}\right) \\
& =\varrho\left(s_{B}\right) \varrho\left(s_{C}\right)-\varrho\left(t_{B}\right) \varrho\left(t_{C}\right)
\end{aligned}
$$

by a comparison inequality (A6) where $\varrho^{\prime}$ denotes the state obtained from $\varrho$ by the interchange of $s$ and $t$ variables ${ }^{4}$.

Since $\varrho\left(s_{B}\right)=\varrho\left(t_{B}\right), \varrho\left(s_{C}\right)=\varrho\left(t_{C}\right)$ by the induction hypothesis, we have

$$
\varrho\left(y_{A}\right)=2^{-\frac{1}{2}|A|} \varrho\left(s_{A}-t_{A}\right)=2^{1-|A|} \sum_{\substack{B \cup C=A \\|C| \text { odd }}} \varrho\left(x_{B} y_{C}\right)=2^{1-|A|} \varrho\left(y_{A}\right)
$$

which implies $\varrho\left(y_{A}\right)=0$.

3 In the summation over subsets of $A$ we distinguish different occurences of the same lattice point (multiplicities). Otherwise combinatorial factors should be included in the expression

4 Note that for $\varrho^{\prime}$ the effective Hamiltonian for the region $\Lambda$ has the form

$$
-H_{A}^{\prime}=\sum_{i, j \in \Lambda} J_{i j}\left(s_{i} s_{j}+t_{i} t_{j}\right)+\sum_{i \in \Lambda} \alpha_{i}^{\prime} s_{i}+\beta_{i}^{\prime} t_{i}
$$

where $\alpha_{i}^{\prime}=\beta_{i}, \beta_{i}^{\prime}=\alpha_{i}$. Since $\alpha_{i} \geqq\left|\beta_{i}\right|$ the hypotheses of comparison Theorem A3 are satisfied 


\section{Uniqueness of the Phase}

We consider the plane rotator in zero external field and suppose there is no spontaneous magnetization. We will show that there is a unique phase. We use the equivalence between phases and tangents to the graph of the pressure [9]. For $f \in \mathfrak{A}$ we consider the perturbation $\lambda f$ to the Hamiltonian $H$. That is, we consider

$$
H_{\lambda}=H-\lambda \sum \tau_{i} f
$$

where we sum over the lattice translates of $f$. (Equilibrium states for $H_{\lambda}$ are defined as in Section 2, via finite volume states with some effective Hamiltonian $H_{\lambda, A}$, or directly by the DLR equations appropriate for $H_{\lambda}$.) If we can show that the pressure $p_{\lambda}$ is differentiable at $\lambda=0$ it follows that all invariant equilibrium states take the same value on $f$. This may be formulated as

4.1. Lemma. Let there exist a sequence of positive numbers $\left(\lambda_{n}\right)_{n \in \mathbb{N}}$ and another one of negative numbers $\left(\lambda_{n}^{\prime}\right)_{n \in \mathbb{N}}$, both converging to zero and invariant equilibrium states $\varrho_{\lambda_{n}^{\prime}}$, $\varrho_{\lambda_{n}}$ of $H_{\lambda_{n^{\prime}}}, H_{\lambda_{n}}$ such that $\lim _{n \rightarrow \infty} \varrho_{\lambda_{n}^{\prime}}(f)=\lim _{n \rightarrow \infty} \varrho_{\lambda_{n}}(f)$.

Then all the $\varrho \in \Delta_{I}(H)$ take the same value on $f\left(=\lim _{n \rightarrow \infty} \varrho_{\lambda_{n}}(f)\right)$.

Proof. The equivalence between invariant equilibrium states and tangents to the graph of the pressure [9], together with the hypothesis and the convexity of $P_{\lambda}$ shows that $P_{\lambda}$ is differentiable at $\lambda=0$ and this is equivalent to the conclusion $[9,13]$.

We shall apply Lemma 4.1 to various perturbations and first to $\left\{s_{A}, t_{A}\right\}$. Once we have shown uniqueness on these functions (Lemma 4.2) we use this result to get uniqueness on the even subspace $\mathfrak{H}_{e}$.

4.2. Lemma. All phases take the same value on $s_{A}$ and on $t_{A}$.

Proof. The proof proceeds in three steps.

a) $\varrho_{s}\left(s_{A}\right)=\varrho_{s}\left(t_{A}\right)$ for all $A$, and $\varrho_{s}\left(s_{A}\right)=0$ if $|A|$ odd :

Since $M=0$ Lemma 4.1 implies $\varrho_{s}\left(s_{i}\right)=0$. Since $\varrho_{s}\left(t_{i}\right)=0$ by construction, we have $\varrho_{s}\left(s_{i}-t_{i}\right)=0$. By the "bootstrap" Theorem 3.4, $\varrho_{s}\left(s_{A}-t_{A}\right)=0$ for all $A$. Since $\varrho_{s}\left(t_{A}\right)=0$ if $|A|$ odd by construction, we may also conclude $\varrho_{s}\left(s_{A}\right)=0$ if $|A|$ odd.

b) For all $\varrho \in \Delta(H) \varrho\left(s_{A}\right)=\varrho\left(t_{A}\right)$ for all $A$ and $\varrho\left(s_{A}\right)=0$ if $|A|$ odd :

By Lemma $3.2 \varrho\left(s_{A}-t_{A}\right) \leqq \varrho_{S}\left(s_{A}-t_{A}\right)=0$ if $|A|$ even [expanding in terms of cosines-Eq. (3.1)]. Thus by the symmetry of interchanging $s$ and $t$ variables, we conclude

$$
\varrho\left(s_{A}-t_{A}\right)=0 \text { for all } \varrho \in \Delta(H),|A| \text { even . }
$$

If $|A|$ odd, $\varrho\left(s_{A}\right) \leqq \varrho_{s}\left(s_{A}\right)=0$. By the symmetry $s \rightarrow-s$, we conclude

$$
\varrho\left(s_{A}\right)=0 \text { for all } \varrho \in \Delta(H),|A| \text { odd . }
$$

By the symmetry of interchanging $s$ and $t$ variables we now conclude

$$
\varrho\left(t_{A}\right)=0 \text { for all } \varrho \in \Delta(H),|A| \text { odd } .
$$


c) All phases take the same value on $s_{A}$ and on $t_{A}$ :

Consider the perturbation $\pm \lambda s_{A}, \lambda>0$.

Let $\varrho_{+\lambda}$ be the (translation invariant) limit (Theorem 3.1) as $\Lambda \nearrow \mathbb{Z}^{d}$ of \langle\rangle$_{s,+\lambda, \Lambda}$ and $\varrho_{+}=\lim _{\lambda>0} \varrho_{+\lambda}$ (Griffiths' inequalities). Let $\varrho_{-\lambda}$ be any weak* limit point as $\Lambda \nearrow \mathbb{Z}^{d}$ of \langle\rangle$_{s,-\lambda, \Lambda}$ and let $\varrho_{-\lambda}$ be an average over translations of $\varrho_{-\lambda}$. Let $\varrho_{-}$be a weak $*$ limit point of $\varrho_{-\lambda}$ as $\lambda \rightarrow 0$. Since by a comparison inequality [Eq. (A4)]

$$
\left\langle s_{B}\right\rangle_{s, \lambda, \Lambda} \geqq\left\langle s_{B}\right\rangle_{s,-\lambda, \Lambda}
$$

it follows that

$$
\varrho_{+}\left(s_{B}\right) \geqq \varrho_{-}\left(s_{B}\right)
$$

Similarly

$$
\varrho_{+}\left(t_{B}\right) \leqq \varrho_{-}\left(t_{B}\right) .
$$

Since $\varrho_{ \pm} \in \Delta_{I}(H)$, it follows by point (b) that $\varrho_{ \pm}\left(s_{B}-t_{B}\right)=0$ and so $\varrho_{+}\left(s_{A}\right)$ $=\varrho_{-}\left(s_{A}\right)$. By Lemma 4.1 all phases agree on $s_{A}$. By the symmetry of interchanging $S$ and $t$ variables all phases agree on $t_{A}$.

4.3. Lemma. All phases take the same value on the even subspace $\mathfrak{\mathfrak { X }}_{e}$.

Proof. a) Consider the perturbation $\pm \lambda r_{A} \cos M \phi$. From a comparison inequality (Theorem A4) we have

$$
\left\langle r_{B} \cos N \phi\right\rangle_{s, \lambda, \Lambda} \geqq\left\langle r_{B} \cos N \phi\right\rangle_{s,-\lambda, \Lambda} \text { for } \lambda>0 .
$$

Taking weak* limit points as in Lemma 4.2 we obtain states $\varrho_{ \pm}$which satisfy

$$
\varrho_{+}\left(r_{B} \cos N \phi\right) \geqq \varrho_{-}\left(r_{B} \cos N \phi\right) .
$$

b) If $|B|$ is even, inequality (4.1) implies

$$
\begin{aligned}
\varrho_{-}\left(s_{A} t_{B}\right) & =\varrho_{-}\left(s_{A}\left(s_{B}+t_{B}\right)\right)-\varrho_{-}\left(s_{A} s_{B}\right) \\
& \leqq \varrho_{+}\left(s_{A}\left(s_{B}+t_{B}\right)\right)-\varrho_{-}\left(s_{A} s_{B}\right)
\end{aligned}
$$

and

$$
\begin{aligned}
\varrho_{-}\left(s_{A} t_{B}\right) & =-\varrho_{-}\left(s_{A}\left(s_{B}-t_{B}\right)\right)+\varrho_{-}\left(s_{A} s_{B}\right) \\
& \geqq-\varrho_{+}\left(s_{A}\left(s_{B}-t_{B}\right)\right)+\varrho_{-}\left(s_{A} s_{B}\right) .
\end{aligned}
$$

Since $\varrho_{+}\left(s_{A} s_{B}\right)=\varrho_{-}\left(s_{A} s_{B}\right)$ by Lemma 4.2 the above inequalities imply

$$
\varrho_{-}\left(s_{A} t_{B}\right) \leqq \varrho_{+}\left(s_{A} t_{B}\right)
$$

and

$$
\varrho_{-}\left(s_{A} t_{B}\right) \geqq \varrho_{+}\left(s_{A} t_{B}\right) .
$$

Thus $\varrho_{-}\left(s_{A} t_{B}\right)=\varrho_{+}\left(s_{A} t_{B}\right)$. In particular $\varrho_{+}\left(r_{A} \cos M \phi\right)=\varrho_{-}\left(r_{A} \cos M \phi\right)$. Therefore all phases agree on the even subspace $\mathfrak{U}_{e}$ by Lemma 4.1.

Proof of Theorem 2.1. From Lemma $4.3 \varrho(f)=\varrho_{s}(f)$ for all $f \in \mathfrak{U}_{e}$, and by construction $\varrho_{s}(f)=0$ for all $f$ in the odd sub-space $\mathfrak{U}_{0}$. If there were a phase $\varrho$ such that, $\varrho(f) \neq 0$ for some $f \in \mathfrak{A}_{0}$, then defining $\varrho^{\prime}$ from $\varrho$ by the transformation 
$\phi_{i} \rightarrow-\phi_{i} \forall i$ we would have a nontrivial decomposition of $\varrho_{s}=\frac{1}{2}\left(\varrho+\varrho^{\prime}\right)$ which contradicts the extremality of $\varrho_{s}$ (Theorem 3.1). Thus $\varrho(f)=0$ for all $\varrho \in \Delta_{I}(H)$ if $f \in \mathfrak{A}_{0}$. Thus $\varrho_{s}$ is the unique phase, and by Theorem 3.1 this phase is an extremal equilibrium state. That $\varrho_{s}$ is also the unique quasi-periodic state follows from the extremality of the unique phase, as in [21, Appendix C].

Proof of Corollary 2.3. If the lattice dimension $d=2$, we know by the theorem of Mermin [17] that $\lim _{h \backslash 0}\langle\boldsymbol{\sigma} \cdot \boldsymbol{n}\rangle_{h \text {,periodic }}=0$ with periodic boundary conditions. This implies that $M=0$ and the corollary follows from Theorem 2.1. If $d=3$ we define $T_{c}$ as the lowest temperature such that $M=0$ for all $T>T_{c}$. $\left(T_{c}\right.$ is finite by the high temperature cluster expansion and $T_{c} \neq 0$ by [6].) Theorem 2.1 implies the uniqueness of the phase for $T>T_{c}$.

\section{Uniqueness of the Equilibrium State}

It will be shown that for sufficiently large external field, the plane rotator with fixed length $(d \lambda=\delta(r-b) d r)$ has a unique equilibrium state. We define two phases $\varrho_{M}$ and $\varrho_{m}$ which suitably bound all equilibrium states. Proving that $\varrho_{M}=\varrho_{m}$ then gives the uniqueness of the equilibrium state. We take the external field interaction

$$
-\sum\left(h_{s} s_{i}+h_{t} t_{i}\right)
$$

for the case $h_{s}=h_{t}=h$. (By rotation covariance the result is true for the external field in an arbitrary direction.)

The effective Hamiltonian for the region $\Lambda$ has the form (3.4) where $\alpha_{i}, \beta_{i}$ depend on the exterior configuration. If $h \geqq b \sum_{j} J_{i j}$ then $\alpha_{i}, \beta_{i} \geqq 0$ for all exterior configurations. This allows the use of correlation inequalities for all equilibrium states. Let

$$
\begin{aligned}
A_{i} & =\max _{\omega \in A_{c}} \alpha_{i}=\max _{\omega \in A_{c}} \beta_{i} \\
B_{i} & =\min _{\omega \in A_{c}} \alpha_{i}=\min _{\omega \in A_{c}} \beta_{i}
\end{aligned}
$$

and define the states \langle\rangle$_{M, A}$ (resp. \langle\rangle$_{m, \Lambda}$ ) by taking $\alpha_{i}=A_{i}, \beta_{i}=B_{i}$ (resp. $\alpha_{i}=B_{i}$, $\beta_{i}=A_{i}$ ).

Note that \langle\rangle$_{m, \Lambda}$ is obtained from \langle\rangle$_{M, A}$ by the interchange of $s$ and $t$ variables. Then from comparison inequalities (A4, A5) for any exterior configuration $\omega$

$$
\begin{aligned}
& 0 \leqq\left\langle s_{B}\right\rangle_{m, \Lambda} \leqq\left\langle s_{B}\right\rangle_{\omega, \Lambda} \leqq\left\langle s_{B}\right\rangle_{M, A} \\
& 0 \leqq\left\langle t_{B}\right\rangle_{M, \Lambda} \leqq\left\langle t_{B}\right\rangle_{\omega, \Lambda} \leqq\left\langle t_{B}\right\rangle_{m, A}
\end{aligned}
$$

and

$$
\left|\left\langle s_{A} t_{B}\right\rangle_{M, A}-\left\langle s_{A} t_{B}\right\rangle_{\omega, A}\right| \leqq\left\langle s_{A}\right\rangle_{M, A}\left\langle t_{B}\right\rangle_{\omega, A}-\left\langle s_{A}\right\rangle_{\omega, A}\left\langle t_{B}\right\rangle_{M, A}
$$

from (A6).

5.1. Lemma. The following infinite volume limits exist.

$$
\varrho_{M} \equiv \lim _{\Lambda \gg \mathbb{Z}^{d}}\langle\rangle_{M, \Lambda}, \quad \varrho_{m} \equiv \lim _{\Lambda>\mathbb{Z}^{d}}\langle\rangle_{m, \Lambda} .
$$


Proof. It follows from Equations (5.1) that ${ }^{5}$

$$
\begin{aligned}
& \left\langle s_{A}\right\rangle_{M, A} \text { decreases as } \Lambda \text { increases, } \\
& \left\langle t_{A}\right\rangle_{M, A} \text { increases as } \Lambda \text { increases. }
\end{aligned}
$$

Thus the limits as $\Lambda \nearrow \mathbb{Z}^{d}$ exist. From (5.2) it now follows that ${ }^{5}\left\langle s_{A} t_{B}\right\rangle_{M, \Lambda}$ converges as $\Lambda \nearrow \mathbb{Z}^{d}$. By the interchange of $s$ and $t$ variables the same holds for \langle\rangle$_{m, \Lambda}$.

Proof of Theorem 2.2. The Lee-Yang theorem for this model has been deduced by Dunlop and Newman [4] from a theorem of Suzuki and Fisher [23]. Although it is not stated in this form in [4], one can deduce from [23] analyticity of the pressure in $h_{s}$ with $h_{t}$ fixed, real, and non-zero and analyticity in $h_{t}$ with $h_{s}$ fixed, real and nonzero. Here $h_{s}$ (resp. $\left.h_{t}\right)$ is the field in the $s$-direction (resp. the $t$-direction). (In our case we are interested in a neighbourhood of $h_{s}=h_{t}=h$.) Therefore for all $h \neq 0, \varrho\left(s_{i}\right)$ and $\varrho\left(t_{i}\right)$ are independent of $\varrho \in \Delta_{I}(H)$.

In particular $\varrho_{M}\left(s_{i}\right)=\varrho_{M}\left(t_{i}\right)$. The "bootstrap" Theorem 3.5 then implies $\varrho_{M}\left(s_{A}\right)$ $=\varrho_{M}\left(t_{A}\right)$ for all $A$. By the symmetry of interchanging $s$ and $t$ variables we conclude that $\varrho_{M}\left(s_{A}\right)=\varrho_{m}\left(s_{A}\right)$. Now by Equations (5.1) we conclude that all equilibrium states take the same value on $s_{A}$; similarly for $t_{A}$. By Equation (5.2) all equilibrium states take the same value on $s_{A} t_{B}$.

\section{Generalizations}

In this section we discuss extensions of results derived in preceding sections.

Using a modification of Condition A, Messager et al. [24] have obtained uniqueness of the phase in any nonzero external field with the Lee-Yang theorem (e.g. fixed length rotator).

Theorems 2.1, 2.2, and Corollary 2.3 extend to long-range interactions with sufficiently rapid fall-off.

For $d=2$, Corollary 2.3 is valid with $J_{i j}$ such that $\sum_{j \in \mathbb{Z}^{2}} J_{i j}|j|^{2}<\infty$ i.e. for $J_{i j}$ decreasing like $|i-j|^{-\alpha}$ with $\alpha>4$. Kunz and Pfister [11] have shown that if $J_{i j}$ decreases like $|i-j|^{-\alpha}$ with $2<\alpha<4$, spontaneous magnetization occurs at low temperatures. They remark that in the borderline case, $\alpha=4$, there is no spontaneous magnetization. The conclusion of Corollary 2.3 should hold in this case too.

Theorem 2.2 is valid for any a priori measure satisfying Condition A and such that the pressure is differentiable in $h_{s}$ and $h_{t}$ (the $s$ - and $t$-components of the external field). In particular a weak version of the Lee-Yang property would be sufficient.

We will denote by "generalized interaction" a Hamiltonian of the form

$$
-H=\sum_{A} J(A)\left(s_{A}+t_{A}\right)
$$

where $J(A) \geqq 0$ and $|A|$ is even.

5 Inequalities (5.1) and (5.2) hold for any exterior configuration and hence for any boundary condition $\mathrm{b}$. Take $\mathrm{b}$ induced by \langle\rangle$_{M, \Lambda^{\prime}}$ where $\Lambda^{\prime} \supset \Lambda$ 
Lemma 3.2 and Theorem 3.1 depend only on Ginibre's inequalities [Theorem A1].

Lemma 3.3 is a general result for states on a separable algebra.

Lemma 4.1 is a general statistical mechanical result.

Given that $\varrho_{s}\left(s_{A}\right)=\varrho_{s}\left(t_{A}\right) \forall A$ and $\varrho_{s}\left(s_{A}\right)=0$ for $|A|$ odd, then Lemma $4.2 \mathrm{~b}$ ) follows for any generalized interaction, c) follows for a translation invariant generalized interaction, as does Lemma 4.3.

Given the correlation inequalities of Dunlop [3], one can extend all the above results to get results on the Heisenberg model and on 4-component models. The correlation inequalities are not sufficient to get uniqueness of the translation invariant equilibrium state on all the correlation functions but only on a subset of those.

It has already been noticed that Griffiths' inequality for rotators (Theorem A2-A3) has some analogy with Lebowitz' inequality [15] for Ising models [3, 1, 22]. In support of this, one may note that using only Lebowitz' inequality (in a similar way to the use of Theorem A3 in this paper), and assuming that the equilibrium states are invariant under the spin-flip symmetry, at zero external field, one can show that the equilibrium state is unique. Of course, in this case, the use of FKG inequalities [5] gives much stronger results $[16,19]$.

\section{Appendix : Correlation Inequalities}

We first recall Ginibre's and (generalized) Griffiths' inequalities :

Theorem A1. (Ginibre [7], Dunlop-Newman [4].) Let

$$
d \mu=Z_{\Lambda}^{-1} \exp \sum_{\substack{\operatorname{supp} A \subset A \\ \operatorname{supp} M \subset A}}\left(J(A, M) r_{A} \cos M \phi\right) \prod_{i \in \Lambda} d \lambda_{i}\left(r_{i}\right) d \phi_{i}
$$

with $J(A, M) \geqq 0$.

$\lambda_{i}\left(r_{i}\right)$ any measure on $R_{+}$of compact support. Then

$$
\left\langle r_{A} \cos M \phi r_{B} \cos N \phi\right\rangle \geqq\left\langle r_{A} \cos M \phi\right\rangle\left\langle r_{B} \cos N \phi\right\rangle \text {. }
$$

Theorem A2. (Generalized Griffiths' inequality [1, 3, 12, 18, 22].) Let

$$
\begin{aligned}
& d \mu=Z_{A}^{-1} \exp \left(\sum_{A \subset A} J_{1}(A) s_{A}+J_{2}(A) t_{A}\right) \prod_{i \in A} d v_{i}\left(s_{i} t_{i}\right) \\
& J_{1}(A) \geqq 0 \\
& J_{2}(A) \geqq 0 . \\
& d v_{i}\left(s_{i} t_{i}\right) \text { satisfy Condition } A \text { and are of compact support. Then } \\
& \left\langle s_{A} s_{B}\right\rangle \geqq\left\langle s_{A}\right\rangle\left\langle s_{B}\right\rangle \\
& 0 \leqq\left\langle s_{A} t_{B}\right\rangle \leqq\left\langle s_{A}\right\rangle\left\langle t_{B}\right\rangle .
\end{aligned}
$$

Proof. Stated in this form, the proof is in $[1,3]$. 
We want to extend these inequalities to some cases where $J_{1}$ or $J_{2}$ are not necessarily positive. This is an extension of a remark of Griffiths [8] and of a recent inequality of Lebowitz [14].

Theorem A3. Let

$$
\begin{aligned}
& d \mu=Z_{\Lambda}^{-1} \exp \left(\sum_{\operatorname{supp} A \subset A} J_{1}(A) s_{A}+J_{2}(A) t_{A}\right) \prod_{i \in \Lambda} d v_{i}\left(s_{i} t_{i}\right) \\
& d \mu^{\prime}=Z_{\Lambda}^{\prime-1} \exp \left(\sum_{\operatorname{supp} A \subset A} J_{1}^{\prime}(A) s_{A}+J_{2}^{\prime}(A) t_{A}\right) \prod_{i \in \Lambda} d v_{i}\left(s_{i} t_{i}\right) .
\end{aligned}
$$

$d v_{i}$ satisfy Condition $A$ and are of compact support.

$$
\begin{aligned}
& \left|J_{1}^{\prime}(A)\right| \leqq J_{1}(A) \\
& \left|J_{2}(A)\right| \leqq J_{2}^{\prime}(A) .
\end{aligned}
$$

Then

(i) $\left|\left\langle s_{A}\right\rangle^{\prime}\right| \leqq\left\langle s_{A}\right\rangle$

(ii) $\left|\left\langle t_{A}\right\rangle\right| \leqq\left\langle t_{A}\right\rangle^{\prime}$

(iii) $\left|\left\langle s_{A} t_{B}\right\rangle-\left\langle s_{A} t_{B}\right\rangle^{\prime}\right| \leqq\left\langle s_{A}\right\rangle\left\langle t_{B}\right\rangle^{\prime}-\left\langle s_{A}\right\rangle^{\prime}\left\langle t_{B}\right\rangle$.

Proof. We use the method of duplicate variables [7]. We consider the integral

$$
\int\left(s_{A}-\varepsilon_{1} s_{A}^{\prime}\right)\left(t_{B}^{\prime}-\varepsilon_{2} t_{B}\right) d \mu\left(\left\{s_{i} t_{i}\right\}\right) d \mu^{\prime}\left(\left\{s_{i}^{\prime} t_{i}^{\prime}\right\}\right)
$$

where $\varepsilon_{i}= \pm 1$. Now

$$
\begin{aligned}
& J_{1}(A) s_{A}+J_{2}(A) t_{A}+J_{1}^{\prime}(A) s_{A}^{\prime}+J_{2}^{\prime}(A) t_{A}^{\prime} \\
& =\frac{1}{2}\left[\left(J_{1}(A)+J_{1}^{\prime}(A)\right)\left(s_{A}+s_{A}^{\prime}\right)+\left(J_{1}(A)-J_{1}^{\prime}(A)\right)\left(s_{A}-s_{A}^{\prime}\right)\right. \\
& \left.\quad+\left(J_{2}^{\prime}(A)+J_{2}(A)\right)\left(t_{A}+t_{A}^{\prime}\right)+\left(J_{2}^{\prime}(A)-J_{2}(A)\right)\left(t_{A}^{\prime}-t_{A}\right)\right] .
\end{aligned}
$$

By hypothesis all the coefficients

$$
\begin{array}{ll}
J_{1}(A)+J_{1}^{\prime}(A), & J_{1}(A)-J_{1}^{\prime}(A) \\
J_{2}^{\prime}(A)+J_{2}(A), & J_{2}^{\prime}(A)-J_{2}(A) \quad \text { are positive. }
\end{array}
$$

We expand the exponential in series and develop in each term the factors $s_{A}+s_{A}^{\prime}$, $s_{A}-s_{A}^{\prime}$, etc. with the iterated formula:

$$
x_{1} x_{2} \pm y_{1} y_{2}=\frac{1}{2}\left(\left(x_{1}+y_{1}\right)\left(x_{2} \pm y_{2}\right)+\left(x_{1}-y_{1}\right)\left(x_{2} \mp y_{2}\right)\right) \text {. }
$$

We get a series with positive coefficients of products of integrals which are all positive by Condition A.

Thus the integral $(*)$ is $\geqq 0$, which gives

$$
\varepsilon_{1}\left\langle s_{A} t_{B}\right\rangle^{\prime}+\varepsilon_{2}\left\langle s_{A} t_{B}\right\rangle \leqq\left\langle s_{A}\right\rangle\left\langle t_{B}\right\rangle^{\prime}+\varepsilon_{1} \varepsilon_{2}\left\langle s_{A}\right\rangle^{\prime}\left\langle t_{B}\right\rangle .
$$

This gives

$$
\left|\left\langle s_{A} t_{B}\right\rangle+\varepsilon\left\langle s_{A} t_{B}\right\rangle^{\prime}\right| \leqq\left\langle s_{A}\right\rangle\left\langle t_{B}\right\rangle^{\prime}+\varepsilon\left\langle s_{A}\right\rangle^{\prime}\left\langle t_{B}\right\rangle
$$

where $\varepsilon= \pm 1$. 
(i) follows taking $B=\phi, \quad \varepsilon= \pm 1$

(ii) follows taking $A=\phi, \quad \varepsilon= \pm 1$

(iii) follows taking $\varepsilon=-1$. have:

Combining this method with Ginibre's method of proving his inequalities, we

Theorem A4. Let

$$
\begin{aligned}
& d \mu=Z_{\Lambda}^{-1} \exp \left(\sum_{\substack{\operatorname{supp} M \subset A \\
\text { supp } A \subset A}} J(A, M) r_{A} \cos M \phi\right) \prod d \lambda_{i}\left(r_{i}\right) d \phi_{i} \\
& d \mu^{\prime}=Z_{\Lambda}^{-1} \exp \left(\sum_{\substack{\operatorname{supp} \mathcal{M} \subset A \\
\operatorname{supp} A \subset A}} J(A, M)^{\prime} r_{A} \cos M \phi\right) \prod d \lambda_{i}\left(r_{i}\right) d \phi_{i} .
\end{aligned}
$$

If for all $M, A J(M, A) \geqq\left|J(M, A)^{\prime}\right|$ then

$$
\left\langle r_{B} \cos N \phi\right\rangle \geqq\left\langle r_{B} \cos N \phi\right\rangle^{\prime} .
$$

Proof.

$$
\int\left(r_{A} \cos M \phi-r_{A}^{\prime} \cos M \phi^{\prime}\right) d \mu\left(\left\{r_{i}, \phi_{i}\right\}\right) d \mu\left(\left\{r_{i}^{\prime} \phi_{i}^{\prime}\right\}\right) \geqq 0
$$

by the same method as above. The inequalities used in the proof of Theorem 3.1 are derived as follows.

Theorem A5. (Generalized Dunlop-Newman inequalities [3].) Let $\varrho$ be a state satisfying Ginibre's inequalities (Theorem A1) and such that $\varrho\left(s_{A} t_{B}\right)=0$ if $|B|$ is odd. Then

$$
\begin{aligned}
& \text { i) }\left|\varrho\left(s_{A} t_{B} S_{C} t_{D}\right)-\varrho\left(s_{A} t_{B}\right) \varrho\left(s_{C} t_{D}\right)\right| \\
& \leqq \varrho\left(s_{A} S_{B} S_{C} S_{D}\right)-\varrho\left(s_{A} S_{B}\right) \varrho\left(s_{C} t_{D}\right) \text { if }|B| \text { is even; } \\
& \text { ii) } \varrho\left(s_{A} t_{B} s_{C} t_{D}\right)^{2} \leqq \varrho\left(s_{A} S_{B} S_{C} S_{D}\right)^{2}-\varrho\left(s_{A} S_{B}\right)^{2} \varrho\left(s_{C} S_{D}\right)^{2} \\
& \text { if }|B| \text { is odd. }
\end{aligned}
$$

Proof. We assume that $|D|$ is even in i) and odd in ii) otherwise the 1.h.s. vanishes.

i) Let $l_{i}= \pm 1$. Using Ginibre's inequalities and Equation (3.1), we have

$$
\varrho\left(s_{A}\left(s_{B}+l_{1} t_{B}\right) s_{C}\left(s_{D}+l_{2} t_{D}\right) \geqq \varrho\left(s_{A}\left(s_{B}+l_{1} t_{B}\right)\right) \varrho\left(s_{C}\left(s_{D}+l_{2} t_{D}\right)\right)\right.
$$

which gives

$$
\begin{aligned}
& -l_{1} l_{2}\left(\varrho\left(s_{A} t_{B} s_{C} t_{D}\right)-\varrho\left(s_{A} t_{B}\right) \varrho\left(s_{C} t_{D}\right)\right) \\
& \leqq \varrho\left(s_{A} s_{B} S_{C} S_{D}\right)-\varrho\left(s_{A} s_{B}\right) \varrho\left(s_{C} S_{D}\right)+l_{1}\left(\varrho\left(s_{A} t_{B} s_{C} s_{D}\right)\right. \\
& \left.-\varrho\left(s_{A} t_{B}\right) \varrho\left(s_{C} S_{D}\right)\right)+l_{2}\left(\varrho\left(s_{A} s_{B} s_{C} t_{D}\right)-\varrho\left(s_{A} s_{B}\right) \varrho\left(s_{C} t_{D}\right)\right) .
\end{aligned}
$$

Take the case $l_{1}=1, l_{2}=1$, add it to the case $l_{1}=-1, l_{2}=-1$ and divide by 2 :

$$
-\left(\varrho\left(s_{A} t_{B} S_{C} t_{D}\right)-\varrho\left(s_{A} t_{B}\right) \varrho\left(s_{C} t_{D}\right)\right) \leqq \varrho\left(s_{A} S_{B} S_{C} S_{D}\right)-\varrho\left(s_{A} S_{B}\right) \varrho\left(s_{C} S_{D}\right) .
$$


With $l_{1}=+1, l_{2}=-1$ added to $l_{1}=-1, l_{2}=+1$, we get the same result with the sign instead of - in front of the 1.h.s. So i) is proved.

ii) We use duplicate variables and apply i). Then

$$
\begin{aligned}
& \left|\varrho\left(s_{A} t_{B} S_{C} t_{D}\right)^{2}-\varrho\left(s_{A} t_{B}\right)^{2} \varrho\left(s_{C} t_{D}\right)^{2}\right| \\
& \leqq \varrho\left(s_{A} s_{B} s_{C} s_{D}\right)^{2}-\varrho\left(s_{A} s_{B}\right)^{2} \varrho\left(s_{C} s_{D}\right)^{2}
\end{aligned}
$$

but $\varrho\left(s_{A} t_{B}\right)=\varrho\left(s_{C} t_{D}\right)=0$ since $|B|,|D|$ are odd.

\section{References}

1. Bricmont,J.: Correlation inequalities for two-component fields. Ann. Soc. Sc. Brux. 90, 245-252 (1976)

2. Dobrushin, R.L., Shlosman,S.B.: Absence of breakdown of continuous symmetry in twodimensional models of statistical physics. Commun. math. Phys. 42, 31-40 (1975)

3. Dunlop,F.: Correlation inequalities for multicomponent rotators. Commun. math. Phys. 49, 247256 (1976)

4. Dunlop,F., Newman, C.M.: Multicomponent field theories and classical rotators. Commun. math. Phys. 44, 223-235 (1974)

5. Fortuin,C.M., Ginibre,J., Kastelyn,P.W.: Correlation inequalities on some partially ordered sets. Commun. math. Phys. 22, 89-103 (1971)

6. Fröhlich,J., Simon, B., Spencer,T.: Infrared bounds, phase transitions and continuous symmetry breaking. Commun. math. Phys. 50, 79-85 (1976)

7. Ginibre,J.: General formulation of Griffiths' inequalities. Commun. math. Phys. 16, 310-328 (1970)

8. Griffiths, R. B.: Phase transitions. In: Statistical mechanics and quantum field theory (Les Houches), pp. 241-279. New York, London, Paris: Gordon and Breach 1970

9. Israël, R.: Thesis, Princeton University

10. Israël, R. B.: High-temperature analyticity in classical lattice systems. Commun. math. Phys. 50, 245-257 (1976)

11. Kunz,H., Pfister,Ch.Ed. : First order phase transitions in the plane rotator ferromagnetic model in two dimensions. Commun. math. Phys. 46, 245-251 (1976)

12. Kunz,H., Pfister,Ch.Ed., Vuillermot,P.A.: Inequalities for some classical spin vector models. Bielefeld preprint

13. Lanford,O.E., Ruelle,D.: Observables at infinity and states with short range correlations in statistical mechanics. Commun. math. Phys. 13, 194-215 (1969)

14. Lebowitz,J.L.: Coexistence of phases in Ising ferromagnets. Preprint

15. Lebowitz,J.L.: GHS and other inequalities. Commun. math. Phys. 35, 87-92 (1974)

16. Lebowitz,J.L., Martin-Löf, A.: On the uniqueness of the equilibrium state for Ising spin systems. Commun. math. Phys. 25, 276-282 (1976)

17. Mermin, N.D. : Absence of ordering in certain classical systems. J. Math. Phys. 6, 1061-1064 (1967)

18. Monroë,J.L.: Correlation inequalities for two-dimensional vector spin system. J. Math. Phys. 16, 1809-1812 (1975)

19. Ruelle,D.: On the use of "small external fields" in the problem of symmetry breakdown in statistical mechanics. Ann. Phys. 69, 364-374 (1972)

20. Ruelle,D.: Statistical mechanics. New York: Benjamin 1969

21. Holsztynski,W., Slawny,J.: Phase transitions in ferromagnetic spin systems at low temperatures. Preprint

22. Sylvester, G.S.: Thesis, Harvard University

23. Suzuki, M., Fisher, M.: Zeros of the partition function for the Heisenberg, Ferroelectric and general Ising models. J. Math. Phys. 12, 235-246 (1971)

24. Messager, A., Miracle,S., Pfister,Ch.E.: to be published

Communicated by E. Lieb

Received March 25, 1977; in revised form July 6, 1977 